\title{
Resonance-enhanced multiphoton ionization (REMPI) spectroscopy of p-chlorofluorobenzene
}

\author{
William D. Tuttle, Adrian M. Gardner, and Timothy G. Wright ${ }^{\mathrm{a}, *}$

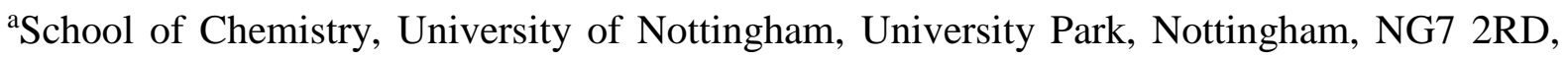 \\ U.K.
}

*To whom correspondence should be addressed. Email: Tim.Wright@ nottingham.ac.uk

\begin{abstract}
The $\mathrm{S}_{1} \leftarrow \mathrm{S}_{0}\left(\tilde{A}^{1} B_{2} \leftarrow \tilde{X}^{1} A_{1}\right)$ electronic transition of para-chlorofluorobenzene has been investigated using resonance-enhanced multiphoton ionization (REMPI) spectroscopy. Assignment of the vibrational structure has been achieved by comparison with corresponding spectra of related molecules, via quantum chemical calculations, and via shifts in bands between the spectra of the ${ }^{35} \mathrm{Cl}$ and ${ }^{37} \mathrm{Cl}$ isotopologues. In addition, we have also partially reassigned a previously-published spectrum of para-dichlorobenzene.
\end{abstract}




\section{Introduction}

In recent work from our group we have focused on the recording and assignment of vibrations of substituted benzenes in the $S_{1}$ state, with a particular interest in identifying common activity across related species. Thus, we have recorded resonance-enhanced multiphoton ionization (REMPI) spectra of the three monohalobenzenes, fluorobenzene (FBz), chlorobenzene $(\mathrm{ClBz})$ and bromobenzene (BrBz) [1],[2],[3], toluene (Tol) [4],[5], para-fluorotoluene ( $p$ FT) [6], [7] and para-xylene (pXyl) [8], [9]. Central to establishing the common activity, is the use of consistent labelling schemes, which we have described for the monosubstituted benzenes [10] and the para-disubstituted benzenes [11]. We shall use the latter scheme in the present work to compare the activity in the titular molecule and other related ones. In contrast to the work on Tol, $p \mathrm{FT}$ and $p \mathrm{Xyl}$ cited above, we shall not report zero-kinetic-energy (ZEKE) photoelectron spectra via intermediate $S_{1}$ vibrational levels as a means of establishing the assignments, but instead rely on quantum chemical calculations of the vibrational wavenumbers, including shifts between $p \mathrm{DFB}$ and $p \mathrm{ClFB}$, shifts between the spectra reported herein for $p^{35} \mathrm{ClFB}$ and $p^{37} \mathrm{ClFB}$, and further comparison to the corresponding spectrum of $p \mathrm{FT}$.

There appears to be very little work on $p \mathrm{ClFB}$, with the available $\mathrm{S}_{0}$ vibrational wavenumbers having been discussed in depth in Ref. 11, to which the reader is referred, and a recommended "best set" put forward; many of these were taken from the gas-phase work of Narasimham et al. [12] and the liquid-phase values of Green [13]. With regards to the $S_{1}$ state, there also appears to be only a handful of studies. The origin transition was analysed via UV absorption spectroscopy by Cvitaš and Hollas [14], but the most pertinent is a resonance-enhanced multiphoton ionization (REMPI) study from Numata et al. [15] to which we shall refer to further in the below; however, we note that only band position wavenumbers were given, but no assignments. There has also been a REMPI study of the $p$ CIFB...methanol complex by Riehn et al. [16], but that of the monomer was not presented.

\section{Experimental}

The vapour above room temperature para-chlorofluorotoluene (98\% purity, Aldrich) was seeded in $\sim 1.5$ bar of Ar and the gaseous mixture passed through a General Valve pulsed nozzle ( $750 \mu \mathrm{m}, 10 \mathrm{~Hz}$, opening time of 180-210 $\mu \mathrm{s}$ ) to create the free jet expansion. The laser was a dye laser (Sirah Cobra-Stretch) operating with either C503 or C540A and was pumped with the third harmonic $(355 \mathrm{~nm})$ of a Surelite Nd:YAG laser, and the fundamental output was frequency doubled using a BBO crystal. The spectra recorded are REMPI spectra, recorded in 
the parent mass channel, with separate spectra recorded for the ${ }^{35} \mathrm{Cl}$ and ${ }^{37} \mathrm{Cl}$ isotopologues. The region below $1400 \mathrm{~cm}^{-1}$ was recorded as a two-colour spectrum, with the ionizing photon being $37990 \mathrm{~cm}^{-1}$, while the remainder of the spectrum was recorded as a one-colour spectrum. This was necessary as it is not possible to ionize via the $\mathrm{S}_{1} \leftarrow \mathrm{S}_{0}$ transition in a (1+1) REMPI process in the first $\sim 350 \mathrm{~cm}^{-1}$ as the ionization energy is too high ${ }^{15}$ (for convenience we continued with the two-colour scheme for the range of the C540A dye employed). Calibration has been achieved by setting the origin band position to that of Cvitaš and Hollas [14], with the uncertainty estimated from the width of the rotational profile.

\section{Calculational Details}

We have shown in the studies cited in the Introduction, that the time-dependent B3LYP (TDB3LYP) method generally appear to work well, with caveats, for calculating vibrational wavenumbers for the $S_{1}$ states of substituted benzenes, and we use that level of theory here. The basis sets employed are aug-cc-pVTZ, which we have also used in previous work and we employed the Gaussian package [17]. We note that the calculated wavenumbers have been scaled by 0.97 to correct both for anharmonicity and other weaknesses in the method; additionally, as we have commented on previously [3], [6], [8] the lowest wavenumber $a_{2}$ vibration ( $D_{14}$ here) does not appear to be described well for substituted benzenes and we do not report values for this vibration, instead relying on the appearance of the spectrum to assign transitions involving this vibration. Further comments on the calculations will be made below.

\section{Results and Assignment}

We present the calculated wavenumbers for $p^{35} \mathrm{ClFB}$ and $p^{37} \mathrm{ClFB}$ in Table 1. It should be noted that the wavenumber ordering of the $D_{i}$ modes in $p \mathrm{ClFB}$ is not the same as in $p \mathrm{DFB}$ and again highlights a weakness in the use of Mulliken labels, even for very closely-related molecules. Additionally, we present the calculated and available experimental values for $p \mathrm{DFB}$ (from previous work [7],[18]) to which we shall compare.

In Figure 1 we show an overview of the REMPI spectra of $p^{35} \mathrm{ClFB}$ together with the corresponding spectra of $p \mathrm{FT}$ and $p \mathrm{DFB}$ for comparison - regions of the latter two spectra have been presented in previous work [6], [7], [8]. The origin of the $S_{1} \leftarrow S_{0}$ transition is measured 
as $36275 \pm 2 \mathrm{~cm}^{-1}$, and is the same for both isotopologues, within $1 \mathrm{~cm}^{-1}$ as judged from the position and band profiles.

To assign the $p$ ClFB spectrum, we compare the activity across the three spectra, and also with the calculated wavenumbers presented in Table 1 , particularly the $p \mathrm{DFB} / p \mathrm{ClFB}$ shifts, but also the $p^{35} \mathrm{ClFB} / p^{37} \mathrm{ClFB}$ isotopic shifts; the latter were very useful in assigning some bands in $\mathrm{ClBz}$ [2]. As is usual, we expect activity from $a_{1}$ symmetry vibrations from the Franck-Condon (FC) principle, and $b_{2}$ vibrations from Herzberg-Teller (HT) vibronic coupling, noting also that the intensity of $a_{1}$ vibrations can also be affected by HT coupling. We give detailed assignments for the region below $1000 \mathrm{~cm}^{-1}$, which are shown in Figure 2 for both the $p^{35} \mathrm{ClFB}$ and $p^{37} \mathrm{CIFB}$ isotopologues, with assignment of the main features also indicated up to $1400 \mathrm{~cm}^{-1}$. We can see that both spectra are very similar with many bands showing no discernible shift, but that there are small but significant shifts in some of the bands to lower wavenumber for the heavier isotopologue. These can be compared to the calculated ${ }^{35} \mathrm{Cl}-{ }^{37} \mathrm{Cl}$ shifts, also presented in Table 1 , and prove a useful tool in confirming the assignments.

Assignment of the spectrum generally turns out to be relatively straightforward with experience gained from our work on similar molecules. For example, the most intense bands are the expected $a_{1}$ fundamentals, but with the caveat that we know that Fermi resonance (FR) can give occasional unexpected activity, while others are HT-allowed $b_{2}$ vibrations. We briefly outline the assignments indicated in Figure 2, noting our rationale when the situation is less clear. Band positions given in the text refer to the ${ }^{35} \mathrm{Cl}$ isotopologue, with the values for both isotopologues being given in Table 2 .

At first sight, assignment of the bands at $344 \mathrm{~cm}^{-1}, 623 \mathrm{~cm}^{-1}$ and $962 \mathrm{~cm}^{-1}$ to the $11^{1}, 10^{1}$ and $8^{1}$ FC-allowed fundamental transitions, respectively, appears straightforward, but we shall come back to the $344 \mathrm{~cm}^{-1}$ band again. We expect the $9^{1}$ transition close to $795 \mathrm{~cm}^{-1}$, but we see that there are two intense bands here. Knowledge of the $9^{1} \ldots .29^{2}$ FR in $p$ FT [7] prompted us to consider whether the same situation could hold here, and indeed since we can confidently identify the $29^{1}$ transition at $397 \mathrm{~cm}^{-1}$, then the $29^{2}$ transition is indeed expected to be close in wavenumber to $9^{1}$. Without further information from time-resolved, coherent-excitation spectroscopies, or zero-kinetic-energy (ZEKE) photoelectron spectroscopy, we cannot comment in much detail on the interaction, but simply represent the transitions by $\left[9^{1} \ldots 2^{2}\right]$, 
with the ordering being unclear. Further comparison to the $p \mathrm{FT}$ and $p \mathrm{DFB}$ spectra, suggest we should expect the $28^{1}$ transition, which can be identified at $546 \mathrm{~cm}^{-1}$, and the $30^{1}$ transition, which we observe at $265 \mathrm{~cm}^{-1}$. The above assignments (barring the $344 \mathrm{~cm}^{-1}$ band - see below) are in very good agreement with the calculated values - See Table 1.

From our [7] and other's [18] previous work, we also expect to observe overtones of various $b_{1}$ vibrations, and it is straightforward to identify the $20^{2}$ transition at $187 \mathrm{~cm}^{-1}$, the $19^{2}$ transition at $488 \mathrm{~cm}^{-1}$ and the $18^{2}$ transition at $861 \mathrm{~cm}^{-1}$. Reaching these latter assignments is more problematic since the calculated $b_{1}$, and $a_{2}$, vibrational wavenumbers are known to be in rather poorer agreement with experiment than are the $a_{1}$ ones [7]. To get a better estimate, we compared the calculated versus experimental wavenumbers for $p$ DFB and applied the same scaling to the $p$ CIFB calculated values for the out-of-plane modes (referred to as " $p$ DFBscaled" values) - this led to a viable assignment for all of the main bands. Assigning the rest of the bands is achieved by using estimations of the fundamentals from the assigned overtones and then looking for FC-allowed $\left(b_{1} \times b_{1}\right)$ or $\left(a_{2} \times a_{2}\right)$ combinations and then also $\left(b_{1} \times a_{2}\right)$ HTallowed combinations. In this way we were able to achieve reasonably confident assignments for most features under $1000 \mathrm{~cm}^{-1}$ with the exceptions being for some very weak bands. We also note that the two bands visible to the red of the main origin band for $p \mathrm{ClFB}$ in Figure 2 at $-31 \mathrm{~cm}^{-1}$ and $-88 \mathrm{~cm}^{-1}$ can be assigned to the $20_{1}^{1}$ and $19{ }_{1}^{1}$ vibrational hot bands. (It is also possible that there is some minor contribution to the $-31 \mathrm{~cm}^{-1}$ band from the $p \mathrm{ClFB}$-Ar complex - a typical shift for such complexes. ${ }^{6}$ )

One feature that we wish to discuss in more detail is the one with a peak at $\sim 344 \mathrm{~cm}^{-1}$. It may be seen that this band has more than one contribution when recorded in the $p^{35} \mathrm{ClFB}$ mass channel, but is apparently a single band in the $p^{37} \mathrm{ClFB}$ mass channel (see Figure 2). For $p^{35} \mathrm{ClFB}$, the two bands are at $344 \mathrm{~cm}^{-1}$ and $347 \mathrm{~cm}^{-1}$, while in $p^{37} \mathrm{ClFB}$ the single band is at $342 \mathrm{~cm}^{-1}$. Based upon the other spectra, we expect to see two transitions at about this wavenumber: $11^{1}$ and $14^{2}$; the $11^{1}$ transition is calculated to shift $\sim 5 \mathrm{~cm}^{-1}$ between the two $\mathrm{Cl}$ isotopologues (see Table 1), while the $14^{2}$ transition is not expected to move significantly. The intensity of the $11^{1}$ transition in the $S_{1} \leftarrow S_{0}$ transition of substituted benzenes appears to be affected by HT-coupling (see, for example, Ref. [8]) and so it is difficult to be sure of its expected intensity, and similar comments could be made about $14^{2}$. However, the observed isotopic shifting of the bands suggests the higher wavenumber, weaker contribution at $~ 347$ 
$\mathrm{cm}^{-1}$ is the $11^{1}$ transition for $p^{35} \mathrm{ClFB}$, and the lower one, whose maximum is uncertain because of the overlap, is due to $14^{2}$. Of course, there is the possibility that $D_{11}$ and $2 D_{14}$ are interacting, but evidence from other studies suggests that this is weak [4],[6],[8]. Since the $D_{11}$ vibration shifts, while the $D_{14}$ overtone is not expected to, it seem that in $p^{37} \mathrm{ClFB}$ these two transitions are now coincident (and the fact that they are also supports the very weak interaction suggested.) Additionally, the fact that the intensity of the band in the $p^{37} \mathrm{ClFB}$ channel is greater than $1 / 3$ of that in the $p^{35} \mathrm{ClFB}$ mass channel is consistent with there being overlapped features.

A summary of the assignments is presented in Table 2 and these are satisfactorily internally self-consistent. In this way we have obtained values for two of the $a_{2}$ fundamentals and all six $b_{1}$ fundamentals, which are summarized in Table 1 . The activity and the wavenumbers are very much in line with activity seen in corresponding spectra of other molecules; in particular, we refer to our recent paper on $p \mathrm{FT}$ where we able to assign all of the $b_{1}$ and $a_{2}$ modes with the aid of ZEKE spectroscopy [7].

We now move onto a more cursory description of the higher wavenumber regions of the spectrum - see Figure 3. We can straightforwardly identify the $6^{1}, 5^{1}$ and $3^{1}$ transitions at 1064 $\mathrm{cm}^{-1}, 1231 \mathrm{~cm}^{-1}$ and $1489 \mathrm{~cm}^{-1}$; these assignments are based on the expected activity together with the good agreement expected between the experimental and calculated wavenumbers. Note that although we did not expect to see either a $4^{1}$ or $7^{1}$ transition, since these are not seen for other molecules. In Figure 3, we have indicated the assignments of the most intense bands moving up to higher wavenumber, and these involve expected progressions in the $D_{11}$ and $D_{9}$ coordinates. We note that the overtone of the latter is complicated by interaction with the $2 D_{29}$ and so, for example we can see a complicated feature at $\sim 1590 \mathrm{~cm}^{-1}$ which will involve the overtones and combination transitions: $9^{2}, 9^{1} 29^{2}$ and $29^{4}$, as well as others that coincidentally fall in this narrow region. Various other complicated features appear throughout the spectrum and if desired assignments can be put forward, but clearly the increasing complexity of bands means that near-coincidences will make firm assignments hard to establish, together with the issues of anharmonicity and associated coupling between like-symmetry levels.

Finally, we have also re-examined the published $S_{1} \leftarrow S_{0}$ spectrum of para-dichlorobenzene ( $p \mathrm{DClB})$, which has been presented in Refs [19], [20] and [21], with an LIF spectrum presented in Ref. [22]. No assignments were presented in Ref. [19], while those given in Ref. [21] are in 
terms of Wilson notation and are not wholly consistent with those of other related molecules, including $p$ DFB [7],[18] and others given herein, in contrast, the assignments in Refs. [21] and [22] are in terms of Mulliken notation (but based on $D_{2 \mathrm{~h}} p \mathrm{DFB}$ ). As a consequence, we recalculated the vibrational wavenumbers using the B3LYP/aug-cc-pVTZ approach and present the findings in Table 1. Notably, optimizing a planar $D_{2 \mathrm{~h}}$ structure gave an out-of-plane imaginary wavenumber - along the $D_{14}$ coordinate - and so we reoptimized a $D_{2}$ symmetry non-planar structure, which led to all-real wavenumbers. (We also then reoptimized the structures of $p \mathrm{DFB}$ and $p \mathrm{ClFB}$, commencing at non-planar structures, but these reverted to the planar structure in both cases.) As we have discussed in Ref. [6], the literature has various comments on the planarity or otherwise of the $S_{1}$ state of various substituted benzenes, with conflicting conclusions from both experiment and theory, but the source of this is not wellestablished and at present, the conclusions from quantum chemical calculations must be treated with caution for the out-of plane modes. In Table 1, we present the calculated wavenumbers for the $D_{2}$ symmetry structure, but keep the same $D_{i}$ vibrational labels, this lowering in symmetry removes the centre of symmetry and can cause mixing of the $D_{i}$ vibrations (see below). (The symmetry classes of the $D_{2 h}$ and $D_{2}$ point groups are related by a removal of the $u / g$ labels for the $D_{2}$ point group.)

In Table 3 we also present the reassignments of the main bands of the $p$ DCIB REMPI spectrum. We take the values reported in ref. [20], as well as estimates for some weaker features seen in their spectrum. As may be seen, there is generally good agreement with the calculated wavenumbers for the $a_{1}$ and $b_{2}$ symmetry modes and those arising from the assignments, which are now consistent with those of the other related molecules. For the $b_{1}$ and $a_{2}$ modes, for the assigned values (except for $D_{14}$ - see above), the agreement with the " $p$ DFB-scaled" values is remarkably good, as noted above for $p \mathrm{ClFB}$. (There are a number of weak features that are not consistent between the different published spectra - it is possible that these may relate to impurities or complexes.) Of note is that in the REMPI spectrum of Ref. [20], some reasonably intense features appear in the range $800-900 \mathrm{~cm}^{-1}$, but these are evident to differing degrees in the REMPI spectrum of Ref. [21] and the LIF spectrum of Ref. [22], with the latter suffering from contributions from the three main isotopologues.

Of particular interest is a band that appears at $1094 \mathrm{~cm}^{-1}$, for which the only reasonable assignment is $10^{2}$. (The fundamental is commented on below as being one of the vibrations this 
is symmetry-allowed in the $C_{2 v}$ asymmetric species, but not in the symmetric $D_{2 h}$ ones.) The assignment of the overtone appears to be clear as it is observed to undergo a significant isotopic shift in the REMPI and LIF studies by the expected amount. The interest lies in the fact that it does not appear to be active to any significant level in other related species, and so its intense appearance is unusual. One rationale may be related to the calculated non-planarity of the $S_{1}$ state of $p \mathrm{DClB}$ - the $D_{10}$ and $D_{13}$ modes are quite close in wavenumber and both become $b_{1}$ symmetry under $D_{2}$ symmetry. These may thus form linear combinations which contain both in-plane and out-of-plane character; thus, the " $D_{10}$ " could gain some FC-activity via this route, since the $13^{2}$ vibration is thought to be active in $p \mathrm{DFB}[18]$ and we believe in $p \mathrm{FT}$ (work in progress). Additionally, an unassigned band at $1139 \mathrm{~cm}^{-1}$ in the LIF spectrum of $p$ DClB [22] looks plausibly assignable to $13^{2}$. These assignments yield vibrational wavenumbers that are in only reasonable agreement with the calculated wavenumbers, but not out of line with those of other related molecules. Further, the large shift in wavenumber upon isotopic substitution is a strong piece of evidence for the $10^{2}$ assignment.

A summary of the deduced assignments is given in Table 3, and the derived fundamental wavenumbers are given in Table 1.

\section{Concluding Remarks}

In the present work, we have assigned the vibrational structure in the $\mathrm{S}_{1} \leftarrow \mathrm{S}_{0}\left(\tilde{A}^{1} B_{2} \leftarrow \tilde{X}^{1} A_{1}\right)$ transition of the $p$ CIFB molecule for the first time. A number of bands below $1100 \mathrm{~cm}^{-1}$ were observed by Numata et al. [15], but no assignments were given therein; these were also seen in the present work, and many more, and are now assigned. We have presented a detailed assignment of the bands below $1000 \mathrm{~cm}^{-1}$, allowing the wavenumber two of the three $a_{2}$ and all six of the $b_{1}$ symmetry vibrations to be established. The values are consistent with those in $p \mathrm{DFB}$, and so support the assignment, despite limitations in the ability of TD-B3LYP to describe these out-of-plane vibrations. We also establish values for the lowest three $b_{2}$ symmetry modes, and those of seven $a_{1}$ modes. The assignments may be seen to be consistent across the spectra of the three molecules presented in Figure 1, with the exception being the low-wavenumber torsional and vibtor transitions in $p \mathrm{FT}$, described in depth in Ref. [6]. There is also good comparison to $p \mathrm{Xyl}$ - see ref. [8], with the torsions having been discussed in depth in Ref. [9]. 
As previously noted in our work on the effectively $C_{2 v}$ molecule, $p$ FT [7], it is interesting to note that the $10^{1}$ and $30^{1}$ transitions are observed, but not in the $D_{2 h} p$ DFB molecule. This is rationalized in terms of symmetry - with the former being FC-allowed and the latter being HTallowed; neither meet the symmetry criteria to be allowed in $p D F B$. It will be noted that in the present work, with the symmetry of $p \mathrm{ClFB}$ being lowered to $C_{2 v}$, then both of these transitions are now observed, as in $p$ FT. We also highlight here the same arguments apply to $6^{1}$, which is an intense band in the REMPI spectra of $p \mathrm{FT}$ and $p \mathrm{ClFB}$, but is absent in that of $p \mathrm{DFB}-$ see Figure 1; additionally, there is no $6^{1}$ band in the $p \mathrm{DClB}$ spectrum (see Ref. [21] and Table 3). However, in all of the spectra there is a $5^{1}$ FC-allowed transition. In Ref. [11] we have discussed the evolution of the $D_{5}$ and $D_{6}$ vibrations on moving from the $D_{2 \mathrm{~h}} p$ DFB molecule to the $C_{2 v}$ asymmetric $p$-dihalobenzenes and other asymmetric para-disubstituted species. Briefly, these evolve from vibrations that contain in-phase $\left(D_{5}\right)$ and out-of-phase $\left(D_{6}\right) \mathrm{C}-\mathrm{F}$ stretches into more local mode $\mathrm{C}-\mathrm{X}$ and $\mathrm{C}-\mathrm{Y}$ stretches, where $\mathrm{X}$ and $\mathrm{Y}$ are the two different para substituents. The change in electronic structure during the $S_{1} \leftarrow S_{0}$ transition causes symmetric changes in the C-F bond lengths in $p \mathrm{DFB}$, and so we only see the $5^{1}$ transition; the same arguments also hold for $p \mathrm{DClB}$. This geometric argument follows the symmetry arguments where the $D_{5}$ vibration is totally symmetric $\left(a_{g}\right)$ in $D_{2 \mathrm{~h}}$, while the $D_{6}$ vibration is of $b_{1 \mathrm{u}}$ symmetry (see Table 1 ). For the asymmetric cases of $p \mathrm{FT}$ and $p \mathrm{ClFB}$ the corresponding bond lengths can change independently and so both $5^{1}$ and $6^{1}$ transitions are active.

We have outlined assignments for the higher wavenumber region of the spectrum, where clear progressions can be identified for totally-symmetric vibrations that correspond to elongation of the benzene ring, in common with the $S_{1} \leftarrow S_{0}$ transition in many substituted benzenes. The level structure becomes increasingly more complicated as the number of levels builds up; as a consequence, the likelihood of vibrational interactions increases and definitive assignment in terms of single combinations/overtones becomes ever less meaningful.

Finally, we have drawn analogies with the published spectra of $p \mathrm{DClB}$, noting a large degree of consistency with the other spectra discussed herein; we have also commented on the apparent anomaly of the intensity of the $10^{2}$ band. 


\section{Acknowledgements}

We are grateful to the EPSRC for funding (grant EP/L021366/1). The EPSRC and the University of Nottingham are thanked for a studentship to W.D.T. We have used the High Performance Computer resource at the University of Nottingham for the quantum chemistry calculations. 


\section{Figure Captions}

Figure 1: Comparison of the first $1380 \mathrm{~cm}^{-1}$ of the REMPI spectra of (a) $p \mathrm{FT}$; (b) $p$ DFB; and (c) $p$ ClFB. The $p$ FT bands marked with an asterisk consist of a number of assignments and will be the subject of future publications.

Figure 2: Assignments of the first $1380 \mathrm{~cm}^{-1}$ of the REMPI spectrum of $p \mathrm{ClFB}$, showing both the $p^{35} \mathrm{ClFB}$ and $p^{37} \mathrm{ClFB}$ isotopologues. Assignments are given for the ${ }^{35} \mathrm{Cl}$ isotopologue and are discussed in the text; those for the ${ }^{37} \mathrm{Cl}$ isotopologue are deduced to be very close or coincident - see text and Table 2.

Figure 3: Overview showing the main progressions of the first $\sim 3200 \mathrm{~cm}^{-1}$ of the REMPI spectrum of $p \mathrm{ClFB}$ - see text. Progressions are indicated with a comb, and we use $\left[9^{1} \ldots 2^{2}\right]$ to indicate a pair of transitions that are thought to be in Fermi resonance (see text), and $\left[9^{1} \ldots 29^{2}\right]^{n}$ to indicate overtones and combinations of the components. The dashed lines indicate the intensity of the frequency-doubled dyes employed, over the indicated ranges. 
Table 1: Calculated and experimental vibrational wavenumbers $\left(\mathrm{cm}^{-1}\right)$ for the $S_{1}$ states of $p \mathrm{ClFB}, p \mathrm{FT}$ and $p \mathrm{DClB}$

\begin{tabular}{|c|c|c|c|c|c|c|c|c|c|c|}
\hline \multirow[t]{2}{*}{$D_{i}^{\mathrm{a}}$} & \multirow[t]{2}{*}{ Mulliken $\left(D_{2 \mathrm{~h}}\right)^{\mathrm{b}}$} & \multicolumn{2}{|c|}{$p^{35} \mathrm{ClFB}$} & \multicolumn{3}{|c|}{$p^{37} \mathrm{ClFB}$} & \multicolumn{2}{|c|}{$p \mathrm{DFB}$} & \multicolumn{2}{|c|}{$p \mathrm{D}^{35} \mathrm{ClB}$} \\
\hline & & Calc $^{c}$ & Expt & Calc $^{\mathrm{c}}$ & $\operatorname{Expt}^{\mathrm{d}}$ & $\Delta_{\text {calc }}{ }^{c}$ & Calc $^{c}$ & $\operatorname{Expt}^{\mathrm{e}}$ & $\mathrm{Calc}^{\mathrm{c}}$ & $\mathrm{Expt}^{\mathrm{f}}$ \\
\hline \multicolumn{11}{|c|}{$a_{1}$} \\
\hline$D_{1}$ & $1\left(a_{g}\right)$ & 3143 & & 3143 & & 0 & 3143 & & 3118 & \\
\hline$D_{2}$ & $10\left(b_{1 u}\right)$ & 3130 & & 3130 & & 0 & 3131 & & 3114 & \\
\hline$D_{3}$ & $2\left(a_{g}\right)$ & 1491 & 1489 & 1491 & 1489 & 0 & 1519 & & 1456 & \\
\hline$D_{4}$ & $11\left(b_{1 u}\right)$ & 1411 & & 1411 & & 0 & 1422 & 1335 & 1395 & \\
\hline$D_{5}$ & $3\left(a_{g}\right) / 4\left(a_{g}\right)^{\mathrm{g}}$ & 1211 & 1231 & 1211 & 1232 & 0 & 1235 & 1251 & 1012 & 1054 \\
\hline$D_{6}$ & $12\left(b_{1 u}\right)$ & 1037 & 1064 & 1036 & 1063 & -1 & 1198 & 1015 & 1009 & \\
\hline$D_{7}$ & $4\left(a_{g}\right) / 3\left(a_{g}\right)^{\mathrm{g}}$ & 1112 & & 1112 & & 0 & 1099 & [1116] & 1124 & 1139 \\
\hline$D_{8}$ & $13\left(b_{1 u}\right)$ & 963 & 962 & 963 & 961 & 0 & 951 & 937 & 972 & \\
\hline$D_{9}$ & $5\left(a_{g}\right)$ & 796 & 793 & 796 & 793 & 0 & 820 & 818 & 727 & 726 \\
\hline$D_{10}$ & $14\left(b_{1 u}\right)$ & 617 & 623 & 614 & 620 & -3 & 710 & [666] & 503 & 547 \\
\hline$D_{11}$ & $6\left(a_{g}\right)$ & 348 & 347 & 343 & 342 & -5 & 414 & 410 & 302 & 298 \\
\hline \multicolumn{11}{|c|}{$a_{2}$} \\
\hline$D_{12}$ & $7\left(a_{u}\right)$ & $532(619)$ & & 532 & & 0 & 501 & 583 & $610(710)$ & 714 \\
\hline$D_{13}$ & $9\left(b_{1 g}\right)$ & $482(534)$ & 538 & 482 & 539 & 0 & 429 & 475 & $531(588)$ & 570 \\
\hline$D_{14}$ & $8\left(a_{u}\right)$ & $-\mathrm{h}$ & 172 & $-{ }^{h}$ & 171 & $-\mathrm{h}$ & $-{ }^{\mathrm{h}}$ & 175 & $-{ }^{\mathrm{h}}$ & 166 \\
\hline \multicolumn{11}{|c|}{$b_{1}$} \\
\hline$D_{15}$ & $15\left(b_{2 g}\right)$ & $696(669)$ & 676 & 696 & 676 & 0 & 697 & 670 & $723(695)$ & \\
\hline$D_{16}$ & $28\left(b_{3 u}\right)$ & $653(606)$ & 611 & 652 & 611 & -1 & 667 & 619 & $658(611)$ & 613 \\
\hline$D_{17}$ & $16\left(b_{2 g}\right)$ & $558(520)$ & 519 & 558 & 519 & 0 & 567 & 528 & $556(518)$ & \\
\hline$D_{18}$ & $29\left(b_{3 u}\right)$ & 486 (437) & 431 & 486 & 430 & 0 & 487 & 438 & $481(433)$ & 436 \\
\hline$D_{19}$ & $17\left(b_{2 g}\right)$ & $254(249)$ & 244 & 254 & 244 & 0 & 279 & 274 & $213(209)$ & 208 \\
\hline$D_{20}$ & $30\left(b_{3 u}\right)$ & $99(96)$ & 94 & 99 & 93 & 0 & 124 & 120 & $70(68)$ & 75 \\
\hline \multicolumn{11}{|c|}{$b_{2}$} \\
\hline$D_{21}$ & $18\left(b_{2 u}\right)$ & 3138 & & 3138 & & 0 & 3139 & & 3120 & \\
\hline$D_{22}$ & $23\left(b_{3 g}\right)$ & 3125 & & 3125 & & 0 & 3126 & & 3109 & \\
\hline$D_{23}$ & $24\left(b_{3 g}\right)$ & 1366 & & 1366 & & 0 & 1407 & [1516] & 1357 & \\
\hline$D_{24}$ & $19\left(b_{2 u}\right) / 20\left(b_{2 u}\right)^{\mathrm{g}}$ & 1303 & & 1303 & & 0 & 1397 & & 1305 & \\
\hline$D_{25}$ & $20\left(b_{2 u}\right) / 19\left(b_{2 u}\right)^{\mathrm{g}}$ & 1408 & & 1408 & & 0 & 1317 & [1591] & 1388 & \\
\hline$D_{26}$ & $25\left(b_{3 g}\right)$ & 1225 & & 1225 & & 0 & 1232 & & 1216 & \\
\hline$D_{27}$ & $21\left(b_{2 u}\right)$ & 1018 & & 1018 & & 0 & 1022 & 1100 & 1028 & \\
\hline$D_{28}$ & $26\left(b_{3 g}\right)$ & 542 & 546 & 542 & 546 & 0 & 553 & 558 & 544 & 536 \\
\hline$D_{29}$ & $27\left(b_{3 g}\right)$ & 394 & 397 & 394 & 397 & 0 & 396 & 403 & 336 & 337 \\
\hline$D_{30}$ & $22\left(b_{2 u}\right)$ & 263 & 265 & 261 & 263 & -2 & 347 & 352 & 226 & 226 \\
\hline
\end{tabular}

${ }^{\text {a }}$ See Ref. [11].

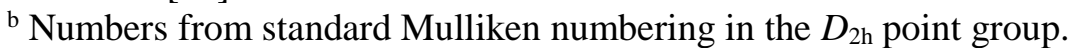

c TD-B3LYP/aug-cc-pVTZ calculated values. Values in parentheses are " $p$ DFB-scaled" values based on the calculated/experimental ratio for $p \mathrm{DFB}$ - see text. $\Delta_{\text {calc }}$ represents the calculated isotopic shift between the ${ }^{35} \mathrm{Cl}$ and ${ }^{37} \mathrm{Cl}$ isotopologues.

d Present work.

e See Refs. [7] and [18]. Values in square brackets are uncertain. Note that the value for $D_{13}$ was concluded in Ref. [7] to be the second offered value in Ref. [18].

${ }^{\mathrm{f}}$ Wavenumber values taken from Refs. [20] and [21]; assignments are from the present work - see text.

${ }^{\mathrm{g}}$ For the indicated vibrations, the energetic ordering changes between $p \mathrm{DFB}$ and $p \mathrm{DClB}$, and hence the "standard" Mulliken label ordering would switch. The first label refers to $p$ DFB and the second to $p \mathrm{DClB}$.

${ }^{\text {h }}$ The $D_{14}$ calculated values are known to be unreliable - see text. 
Table 2: Assignments of the REMPI bands of $p^{35} \mathrm{ClFB}$ and $p^{37} \mathrm{ClFB}{ }^{\text {a }}$

\begin{tabular}{|c|c|c|c|c|c|}
\hline \multicolumn{2}{|c|}{ Position / $\mathrm{cm}^{-1}$} & \multirow{2}{*}{ Assignment } & \multicolumn{2}{|c|}{ Position / $\mathbf{c m}^{-1}$} & \multirow{2}{*}{ Assignment } \\
\hline${ }^{35} \mathrm{Cl}$ & ${ }^{37} \mathrm{Cl}$ & & ${ }^{35} \mathrm{Cl}$ & ${ }^{37} \mathrm{Cl}$ & \\
\hline-88 & -88 & $19_{1}^{1}$ & $1130-1143$ & $1135-1147$ & $\begin{array}{l}{\left[9^{1} \ldots 29^{2}\right]+14^{2},} \\
{\left[9^{1} \ldots 29^{2}\right]+11^{1},}\end{array}$ \\
\hline-31 & -30 & $20_{1}^{1}$ & $1231^{\mathrm{d}}$ & $1232^{\mathrm{d}}$ & $5^{1}$ \\
\hline 0 & 0 & $0^{0}$ & 1245 & 1241 & $10^{2}$ \\
\hline 187 & 186 & $20^{2}$ & 1489 & 1489 & $3^{1}$ \\
\hline 265 & 263 & $30^{1} / 14^{1} 20^{1}$ & $1591^{\mathrm{e}}$ & $1590^{\mathrm{e}}$ & {$\left[9^{1} \ldots 29^{2}\right]^{2 \mathrm{c}}$} \\
\hline 316 & & & $1855^{\mathrm{d}}$ & $1854^{\mathrm{d}}$ & {$\left[9^{1} \ldots 29^{2}\right]+6^{1}$} \\
\hline 337 & 336 & $19^{1} 20^{1}$ & $1860^{\mathrm{d}}$ & $1858^{\mathrm{d}}$ & {$\left[9^{1} \ldots .29^{2}\right]+6^{1}$} \\
\hline 344 & $342^{\mathrm{b}}$ & $14^{2}$ & 2025 & 2025 & $5^{1} 9^{1}$ \\
\hline 347 & $342^{\mathrm{b}}$ & $11^{1}$ & 2038 & 2033 & $9^{1} 10^{2}$ \\
\hline 397 & 397 & $29^{1}$ & 2124 & 2121 & $6^{2}$ \\
\hline 458 & & $14^{1} 20^{3} / 20^{2} 30^{1}$ & 2293 & 2293 & $5^{1} 6^{1}$ \\
\hline 488 & 488 & $19^{2}$ & 2306 & 2300 & $6^{1} 10^{2}$ \\
\hline 502 & & & $2382^{\mathrm{e}}$ & $2382^{\mathrm{e}}$ & {$\left[9^{1} \ldots 29^{2}\right]^{3 c}$} \\
\hline 517 & & $18^{1} 20^{1}$ & $2455^{\mathrm{d}}$ & $2453^{d}$ & $5^{2}$ \\
\hline 546 & 546 & $28^{1}$ & 2469 & 2463 & $6^{2} 11^{1}$ \\
\hline 593 & & & 2477 & $\mathrm{~d}$ & $5^{1} 10^{2}$ \\
\hline 612 & 612 & $17^{1} 20^{1}$ & 2491 & $\mathrm{~d}$ & $10^{4}$ \\
\hline 623 & 620 & $10^{1}$ & $2651^{\mathrm{e}}$ & $2650^{\mathrm{e}}$ & {$\left[9^{1} \ldots 29^{2}\right]^{2}+6^{1 c}$} \\
\hline 689 & 683 & $11^{2} / 11^{1} 14^{2}$ & $2818^{\mathrm{d}, \mathrm{e}}$ & $2817^{\mathrm{d}, \mathrm{e}}$ & {$\left[9^{1} \ldots 29^{2}\right]^{2}+5^{1 b}$} \\
\hline 705 & 704 & $16^{1} 20^{1}$ & $2831^{\mathrm{d}, \mathrm{e}}$ & $2826^{\mathrm{d}, \mathrm{e}}$ & {$\left[9^{1} \ldots 29^{2}\right]^{2}+10^{2 c}$} \\
\hline 710 & 710 & $13^{1} 14^{1}$ & $2911-2919^{d}$ & $2909-2917^{\mathrm{d}}$ & {$\left[9^{1} \ldots 29^{2}\right]+6^{2 c}$} \\
\hline 732 & 731 & & $3083-3088^{d}$ & $3080-3086^{\mathrm{d}}$ & {$\left[9^{1} \ldots 2^{2}\right]+5^{1} 6^{1 c}$} \\
\hline 740 & 738 & $11^{1} 29^{1}$ & $3096-3102^{\mathrm{d}}$ & $\mathrm{d}$ & {$\left[9^{1} \ldots 29^{2}\right]+6^{1} 10^{2 b}$} \\
\hline 762 & 762 & $17^{1} 19^{1}$ & $3178^{\mathrm{e}}$ & & {$\left[9^{1} \ldots 29^{2}\right]^{4 \mathrm{c}}$} \\
\hline 769 & 769 & $15^{1} 20^{1}$ & & & \\
\hline 793 & 793 & {$\left[9^{1} \ldots 29^{2}\right]^{\mathrm{c}}$} & & & \\
\hline 798 & 798 & {$\left[9^{1} \ldots 29^{2}\right]^{\mathrm{c}}$} & & & \\
\hline 808 & 808 & & & & \\
\hline 813 & 811 & $10^{1} 20^{2} / 28^{1} 30^{1}$ & & & \\
\hline 861 & 860 & $18^{2}$ & & & \\
\hline 872 & 866 & $11^{2} 20^{2}$ & & & \\
\hline 887 & $887^{\mathrm{c}}$ & $19^{2} 29^{1} / 14^{2} 28^{1}$ & & & \\
\hline 893 & $887^{\mathrm{c}}$ & $11^{1} 28^{1}$ & & & \\
\hline 936 & 936 & & & & \\
\hline 945 & 944 & $17^{1} 18^{1} / 28^{1} 29^{1}$ & & & \\
\hline 962 & 961 & $8^{1}$ & & & \\
\hline 975 & 975 & $9^{1} 20^{2}$ & & & \\
\hline 982 & 981 & $29^{2} 20^{2}$ & & & \\
\hline 1064 & 1063 & $6^{1}$ & & & \\
\hline
\end{tabular}

${ }^{a}$ Line positions are relative to the origin at $36275 \pm 2 \mathrm{~cm}^{-1}$ (same for both isotopologues). The lower state is the zero point level in $\mathrm{S}_{0}$, unless noted otherwise. Detailed assignments are given in the range $-100-1000 \mathrm{~cm}^{-1}$, with selected assignments given for the higher wavenumber bands. See Figures 2 and 3 for the spectra. Below $1000 \mathrm{~cm}^{-1}$, if an assignment is not given, no reasonable assignment could be found - such bands are generally very weak. If two transitions are given separated by a solidus, then these are thought to be overlapping transitions. Some 
bands were too weak to be definitively identified for the ${ }^{37} \mathrm{Cl}$ isotopologue and the Position entry is left blank for these.

${ }^{\mathrm{b}}$ Bands are coincident.

${ }^{c}$ Bands involving the $9^{1}$ and $29^{2}$ transitions, and combinations and overtones thereof, arise from eigenstates that are linear combinations of $D_{9}$ and $2 D_{29}$, but the precise make-up is unknown. Such bands are indicated by square brackets for the most intense feature of a group, but this is usually part of a more complicated feature, whose eigenstates will be mixtures of the indicated $S_{1}$ levels that are involved in the transition. No inference should be made from the ordering of the transitions given as to the relative magnitudes of the contributing states.

${ }^{\mathrm{d}}$ Overlapped feature. Indicated position or range, if given, is a best estimate.

${ }^{\mathrm{e}}$ Band position taken from the maximum of a clump of bands, which are thought to arise from transitions to levels that comprise various combinations of the indicated transitions. 
Table 3: Assignments of the REMPI/LIF bands of $p \mathrm{D}^{35} \mathrm{ClB}$

\begin{tabular}{|c|c|c|}
\hline Position $^{a}$ & $\begin{array}{c}\text { Assignment } \\
D_{i} \mathrm{~b} \\
\end{array}$ & $\begin{array}{c}\text { Previous Assignment } \\
\text { Mulliken }-D_{2 h} / \text { Wilson }\end{array}$ \\
\hline \multicolumn{3}{|r|}{ 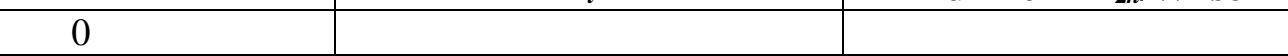 } \\
\hline 150 & $20^{2}$ & $30^{2}\left(11^{1}\right)$ \\
\hline 245 & $14^{1} 20^{1}$ & - \\
\hline 301 & $11^{1}$ & $6^{1}\left(7 a^{1}\right)$ \\
\hline 334 & $14^{2}$ & $8^{2}\left(16 a^{2}\right)$ \\
\hline 339 & $29^{1}$ & $27^{1}\left(9 b^{1}\right)$ \\
\hline 416 & $19^{2}$ & \\
\hline 451 & $30^{2}\left(+11^{1} 20^{2}\right)^{d}$ & $17^{2}\left(10 b^{2}\right)$ \\
\hline 538 & $28^{1}$ & $26^{1}\left(6 b^{1}\right)$ \\
\hline 588 & $14^{1} 18^{1}$ & $29^{2}\left(16 b^{2}\right)$ \\
\hline 602 & $11^{2}$ & \\
\hline 636 & $11^{1} 14^{2}$ & \\
\hline 641 & $11^{1} 29^{1}$ & $6^{1} 27^{1}\left(7 a^{1} 6 b^{1}\right)$ \\
\hline 672 & $14^{2} 29^{1}$ & $8^{2} 27^{1}\left(16 a^{2} 9 b^{1}\right)$ \\
\hline 680 & $29^{2}$ & \\
\hline 688 & $16^{1} 20^{1}$ & \\
\hline 708 & $A r^{\mathrm{e}}$ & \\
\hline 716 & $A r^{\mathrm{e}}$ & \\
\hline 727 & $9^{1}\left(+17^{1} 19^{1}\right)^{d}$ & $5^{1}\left(6 a^{1}\right)$ \\
\hline 831 & $19^{4}$ & $16^{2}\left(4^{2}\right)$ \\
\hline $838 / 841^{f}$ & $11^{1} 28^{1}$ & $6^{1} 26^{1}\left(7 a^{1} 6 b^{1}\right)$ \\
\hline 870 & $18^{2}$ & \\
\hline 877 & $12^{1} 14^{1}$ & \\
\hline 1027 & $9^{1} 11^{1}$ & $5^{1} 6^{1}\left(7 a^{1} 6 a^{1}\right)$ \\
\hline 1053 & $5^{1}$ & $4^{1}\left(1^{1}\right)$ \\
\hline 1059 & $9^{1} 14^{2}$ & \\
\hline 1065 & $9^{1} 29^{1}$ & $27^{1} 5^{1}\left(9 b^{1} 6 a^{1}\right)$ \\
\hline 1079 & $28^{2}$ & \\
\hline 1094 & $10^{2}$ & $14^{2}\left(20 a^{2}\right)$ \\
\hline 1139 & $13^{2}$ & $3^{1}\left(9 a^{1}\right)$ \\
\hline
\end{tabular}

${ }^{a}$ From Ref. [20] and [21] unless otherwise stated; italicized values have been estimated from the spectra presented in those studies.

$\mathrm{b}$ This work - see text.

${ }^{c}$ Assignments from Ref. [20], also carried over into Refs. [21] and [22]; note that the $D_{2 h}$ Mulliken labels are termed "Herzberg" labels in Ref. [21]. An empty entry means an assignment was not given in previous work.

${ }^{\mathrm{d}}$ Possible coincident band with minor contribution in parentheses.

e These appear to be bands arising from complexes of $p$ DClB with Ar, from the work of Ref. [21].

${ }^{\mathrm{f}}$ From Ref. [22]. 
Figure 1

(a) $p \mathrm{FT}$ 


\section{Figure 2}

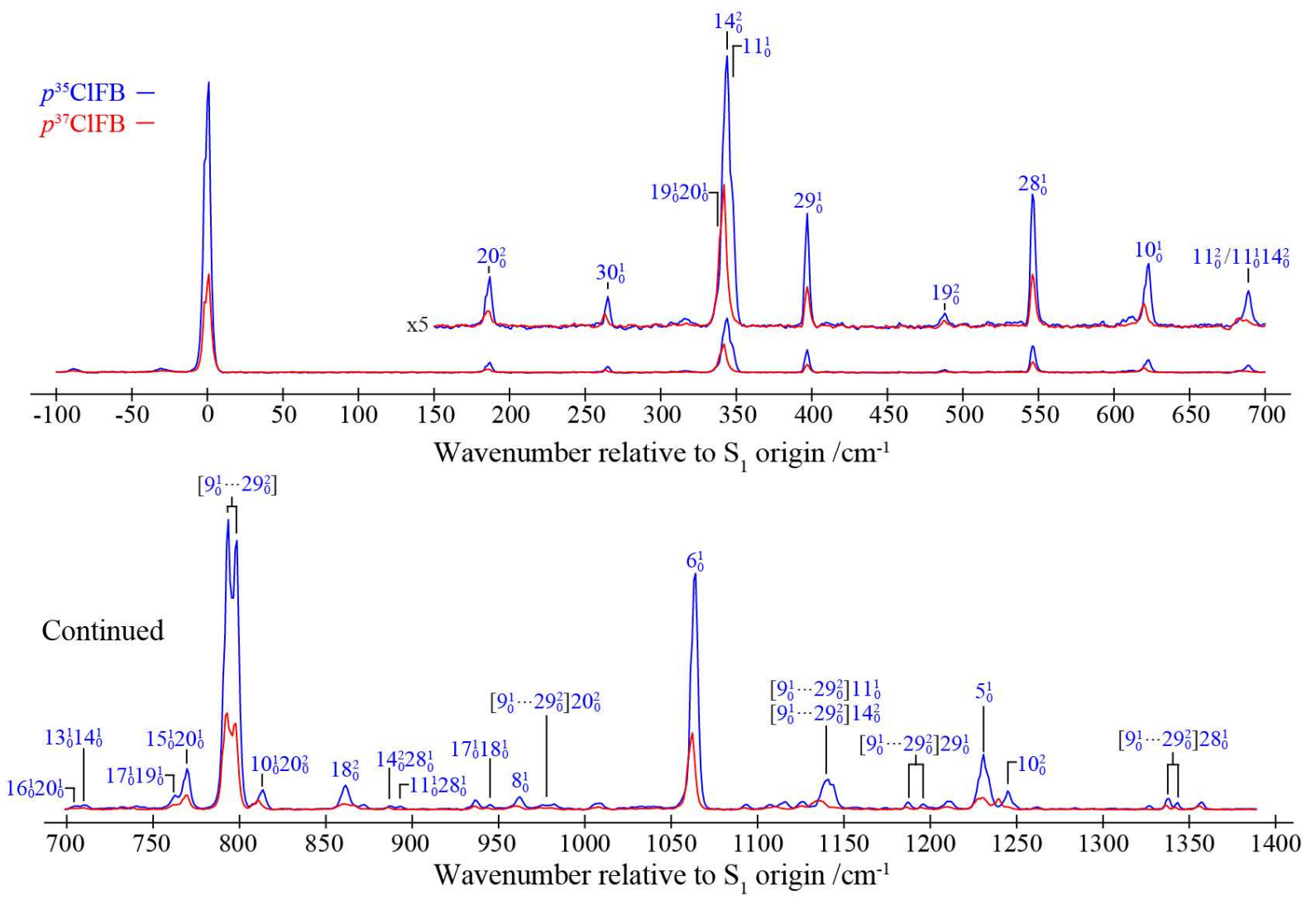


Figure 3

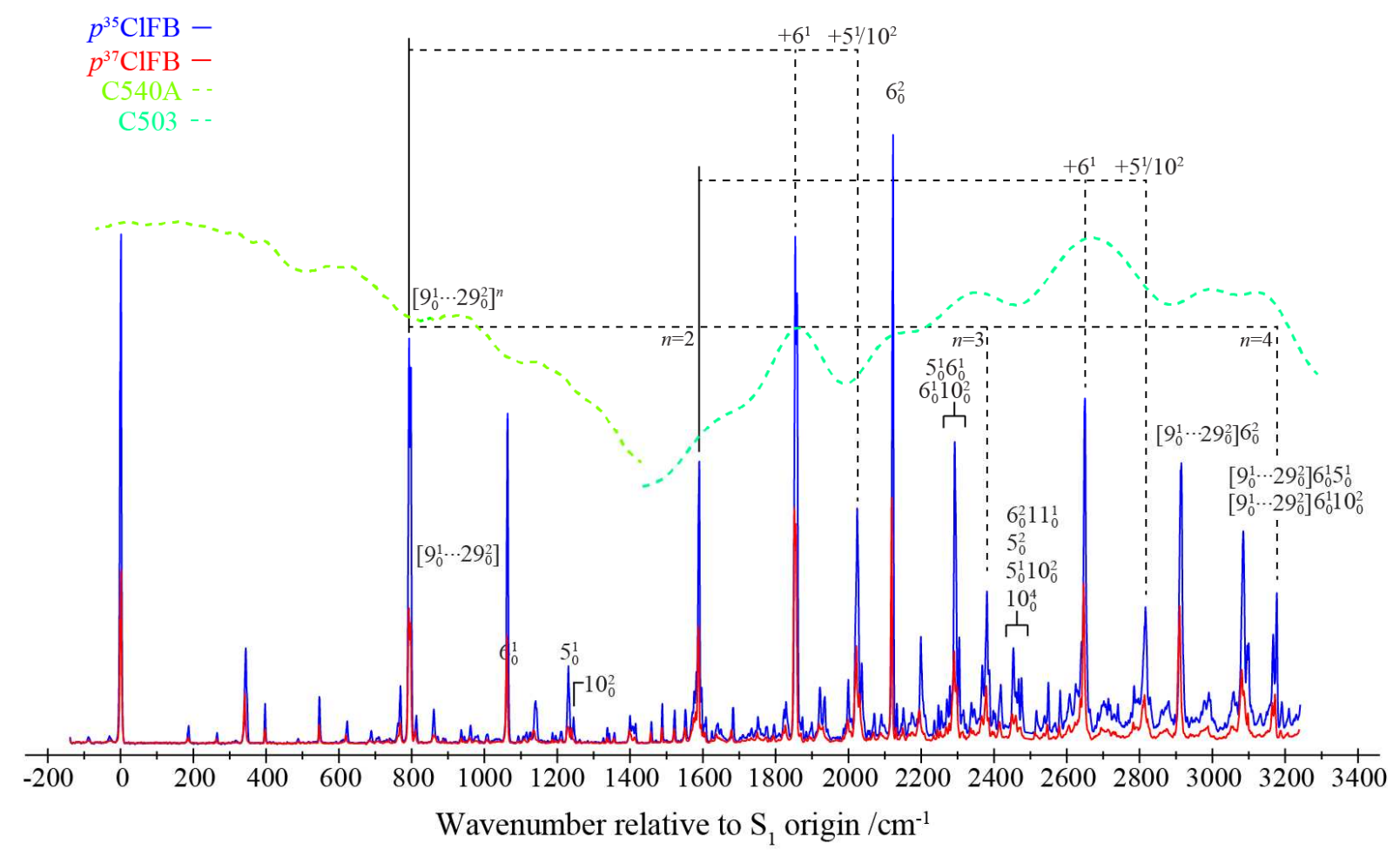




\section{References}

${ }^{1}$ J. P. Harris, A. Andrejeva, W. D. Tuttle, I. Pugliesi, C. Schriever, T. G. Wright, J. Chem. Phys. 141 (2014) 244315.

${ }^{2}$ A. Andrejeva, W. D. Tuttle, J. P. Harris, T. G. Wright, J. Chem. Phys. 143 (2015) 104312.

${ }^{3}$ A. Andrejeva, W. D. Tuttle, J. P. Harris, T. G. Wright, J. Chem. Phys. 143 (2015) 244320.

${ }^{4}$ A. M. Gardner, A. M. Green, V M. Tamé-Reyes, V. H. K. Wilton, T. G. Wright

J. Chem. Phys. 138 (2013) 134303.

${ }^{5}$ A. M. Gardner, A. M. Green, V. M. Tamé-Reyes, K. L. Reid, J. A. Davies, V. H. K. Parkes, T. G. Wright J. Chem. Phys. 140 (2014) 114038.

${ }^{6}$ A. M Gardner, W. D. Tuttle, L. Whalley, A. Claydon, J. H. Carter, T G. Wright, J. Chem. Phys. 145 (2016) 124307.

${ }^{7}$ W. D. Tuttle, A. M. Gardner, L. E. Whalley, T. G. Wright, J. Chem. Phys. 146 (217), 244310.

${ }^{8}$ W. D. Tuttle, A. M Gardner, K. B. O’Regan, W. Malewicz, T. G. Wright, J. Chem. Phys. 146 (2017) 124309.

${ }^{9}$ A. M. Gardner, W. D. Tuttle, P. Groner, T. G. Wright, J. Chem. Phys. 146 (2017) 124308.

${ }^{10}$ A. M. Gardner, T. G. Wright. J. Chem. Phys. 135 (2011) 114305.

${ }^{11}$ A. Andrejeva, A. M. Gardner, W. D. Tuttle, T. G. Wright, J. Molec. Spectrosc. 321 (2016) 28.

${ }^{12}$ N. A. Narasimham, M. Z. El-Sabban, J. R. Nielsen, J. Chem. Phys. 24 (1956) 420.

${ }^{13}$ J. H. S. Green, Spectrochim. Acta 26A (1970) 1503.

${ }^{14}$ T. Cvitaš, J. M. Hollas, Molec. Phys. 18 (1970) 261.

${ }^{15}$ Y. Numata, Y. Ishii, M. Watahiki, I. Suzuka, M. Ito, J. Phys. Chem. 97 (1993) 4930.

${ }^{16}$ C. Riehn, K. Buchhold, B. Reimann, S. Dafari, H.-D. Barth, B. Brutschy, P. Tarakeshwar, K. S.

Kim, J. Chem. Phys. 112 (2000) 1170.

${ }^{17}$ Gaussian 09, Revision E.01, M. J. Frisch et al. Gaussian, Inc., Wallingford CT, 2016.

${ }^{18}$ A.E.W. Knight, S. H. Kable, J. Chem. Phys. 89 (1988) 7139.

${ }^{19}$ R. Tembreull, C. H. Sin, P. Li, H. M. Pang, D. M Lubman, Anal. Chem. 57 (1985), 1186.

${ }^{20}$ E. A. Rohlfing, C. M. Rohlfing, J. Phys. Chem. 93 (1989), 94.

${ }^{21}$ A. Gaber, M. Riese, J. Grotemeyer, J. Phys. Chem. A 112 (2008) 425.

${ }^{22}$ W. D. Sands, R. Moore, J. Phys. Chem. 93 (1989), 101. 